\title{
Medaka spalt acts as a target gene of hedgehog signaling
}

\author{
Reinhard Köster ${ }^{1}$, Reimer Stick ${ }^{2}$, Felix Loosli ${ }^{1}$ and Joachim Wittbrodt ${ }^{1, *}$ \\ 1SFB 271 Junior Group, c/o Max-Planck Institute for Biophysical Chemistry, Am Fassberg, 37077 Göttingen Germany \\ ${ }^{2}$ Institut für Biochemie und Molekulare Zellbiologie, Universität Göttingen, Humboldtallee 23, 37073 Göttingen Germany \\ *Author for correspondence (e-mail: jwittbr@gwdg.de)
}

\section{SUMMARY}

In vertebrates, pattern formation in the eye, central nervous system, somites, and limb depends on hedgehog activity, but a general target gene controlled by hedgehog in all these signaling centers has remained largely elusive. The medaka fish gene spalt encodes a zinc-finger transcription factor, which is expressed in all known hedgehog signaling centers of the embryo and in the organizer region at the midbrain-hindbrain boundary. We show that the spalt expression domains expand in response to ectopic hedgehog activity and narrow in the presence of protein kinase A activity, an antagonist of hedgehog signaling, indicating that spalt is a hedgehog target gene. Our results also suggest a signaling mechanism for anterior-posterior patterning of the vertebrate brain that controls spalt expression at the midbrain-hindbrain boundary in a protein kinase $A$ dependent manner likely to involve an unknown member of the hedgehog family.

Key words: spalt, hedgehog target gene, medaka, patterning, midhindbrain boundary

\section{INTRODUCTION}

Pattern formation in multicellular organisms depends on positional information generated by transient gradients of secreted signaling molecules (reviewed by Lawrence and Struhl, 1996). This graded activity is then permanently established by the differential expression of target genes. In Drosophila, one of the diffusible patterning activities is encoded by the segment polarity gene hedgehog $(h h)$ which mediates pattern formation during segmentation (Lee et al., 1992) and during imaginal disc determination (Tabata and Kornberg, 1994).

Recently several homologues of the Drosophila hedgehog gene have been identified in various vertebrate species, revealing the existence of a hedgehog gene family of secreted signaling molecules (Zardoya et al., 1996). Best characterized is sonic hedgehog (shh), whose spatiotemporal expression is conserved throughout vertebrates. Initially, shh is expressed in the embryonic shield or node during gastrulation, then becomes localized to the ventral midline during axis formation and subsequently to the notochord, floorplate and anteriorly to the prechordal mesoderm. Later shh expression is found in the outgrowing posterior distal limb and the excretory system (reviewed by Hammerschmidt et al., 1997).

Diffusible HEDGEHOG protein emanates from localized sources, referred to as $h h$ signaling centers, and mediates pattern formation over short and long distances. The eyes, the neural tube, the somites, the excretory system and limbs are structures that are organized through the patterning activity of SHH. Patterning in the vertebrate eyes is mediated by SHH emanating from the underlying prechordal mesoderm in the embryonic midline. The finding that shh deficient mice suffer phenotypically from cyclopia (Chiang et al., 1996) indicates that midline signaling by $\mathrm{SHH}$ is necessary for a proper separation of the anterior eye domain. Consistently, cyclopia was also observed when midline signaling was antagonized by overexpression of protein kinase A (PKA), which efficiently inhibits signaling of several members of the hedgehog family (Hammerschmidt et al., 1996). Conversely, ectopic overexpression of shh leads to the expansion of proximal eye structures at the expense of distal structures as shown by the expansion of proximal Pax2 expression and suppression of the distal eye marker Pax6 (Ekker et al., 1995b; Macdonald et al., 1995). In the notochord, shh expression leads to the contact dependent induction of floorplate cells in the neural tube which themselves start to secret $\mathrm{SHH}$ and subsequently induce the differentiation of motor neurons (Ericson et al., 1996 and references therein). In addition, lateral diffusion of $\mathrm{SHH}$ from midline structures was shown to control the patterning of the paraxial mesoderm (Johnson et al., 1994; Fan and Tessier-Lavigne, 1994; Fan et al., 1995). In the excretory system $s h$ is expressed at different sites (Bitgood and McMahon, 1995) and mediates patterning processes and induction events which are currently analyzed in shh deficient mice (Chiang et al., 1996). Finally, during limb development SHH is generated in the posterior part of the outgrowing limbs, the zone-of-polarizing activity (ZPA), which mediates anteroposterior (AP) polarity of the limb. An ectopic source of diffusible $\mathrm{SHH}$ in the anterior part of the limb bud mimics a ZPA and leads to mirror-image duplication of digits (Riddle et al., 1993; Chang, 1994; López-Martinez et al., 1995).

Although the important role of $s h$ in patterning processes of vertebrate development is well established, little is known about the transmission of the HEDGEHOG signal. Recently, the transmembrane protein PATCHED (PTC) was discovered to function as $\mathrm{SHH}$ receptor releasing the bound 
SMOOTHENED (SMO) protein from its inactive state by binding to SHH (Stone et al., 1996; Marigo et al., 1996a). General target genes of $\mathrm{HH}$ that mediate $h h$ signaling at the level of transcription, and are activated in all $h h$ signaling centers, remain largely elusive.

Here we describe the cloning of such a target gene, the medaka (Oryzias latipes) homologue of the Drosophila zinc finger transcription factor spalt (sal). In the Drosophila wing imaginal disc sal was shown to be activated by $h h$ expression (Sturtevant et al., 1997) and to mediate patterning (de Celis et al., 1996; Sturtevant et al., 1997). In agreement with this, we find medaka spalt ( $\mathrm{sal}$ ) expressed in all $h h$ signaling centers and in the organizer at the mid-hindbrain boundary (MHB). Medaka sal is activated by ectopic SHH activity. Suppression of $h h$ signaling by overexpression of protein kinase A (PKA) results in suppression of sal expression. Our results indicate that sal is a $h h$ target gene which is positively regulated by $\mathrm{HH}$ activity in all known signaling centers. In addition, the sal expression domain at the MHB expands in response to ectopic $\mathrm{HH}$ and it narrows in response to PKA activity. Our results are consistent with the proposal that AP patterning of the dorsal vertebrate brain is mediated by $h h$ signaling, although a corresponding member of the $\mathrm{HH}$ family that is expressed in the relevant region of the brain has not yet been identified.

\section{MATERIALS AND METHODS}

\section{Medaka stocks}

Fish were maintained in a constant recirculating system at $28^{\circ} \mathrm{C}$ on a 14 hours light/10 hours dark cycle. Wild-type Oryzias latipes from a closed stock were originally obtained from Carolina Biological, North Carolina, USA. Pairwise mating was performed and collected embryos were kept at $28^{\circ} \mathrm{C}$.

\section{Isolation and sequencing of medaka sal, Pax2 and shh}

Medaka sal was isolated by PCR using degenerate oligonucleotides [5': TGYGCNGARTTYTTYAARTGGAC, 3': CTDATCATYTTCATYTGRTTYTC, 40 cycles: $94^{\circ} \mathrm{C}, 1$ minute (first: 3 minutes); $49^{\circ} \mathrm{C}, 1.5$ minutes; $72^{\circ} \mathrm{C}, 1.5$ minutes (final 10 minutes)] specific for conserved regions of the known spalt genes (Hollemann et al., 1996; Ott et al., 1996). A $2.5 \mathrm{~kb}$ fragment of coding region was amplified from genomic DNA and subsequently used to screen a neurula stage (stage 18) cDNA library (stages refer to Iwamatsu, 1994). The longest clone contained a $4.5 \mathrm{~kb}$ cDNA insert which was sequenced with internal primers (Applied Biosystems 373A DNA Sequencer). The coding region of this cDNA clone could be extended in the $5^{\prime}$ direction by sequencing genomic DNA (accession \#U77376).

A 900 bp medaka Pax2 fragment [5' GCIGAYCCITTYWSIGCIATGCA, 3': GGRTTISWRAAICKCCAIGCYTCRTTRTA, 35 cycles: $94^{\circ} \mathrm{C}, 1$ minute (first: 5 minutes); $5 \times 48^{\circ} \mathrm{C}+30 \times 53^{\circ} \mathrm{C}, 2$ minutes; $72^{\circ} \mathrm{C}, 4$ minutes (final 9 minutes)] and a 297 bp medaka shh fragment [5': CCIAAYTAYAAYCCIGAYATHATITTYAA, 3': YTCRTARTAIACCCARTCRAAICCIG, 35 cycles: $94^{\circ} \mathrm{C}, 1$ minute (first: 5 minutes); $5 \times 48^{\circ} \mathrm{C}+30 \times 53^{\circ} \mathrm{C}, 2$ minutes; $72^{\circ} \mathrm{C}, 4$ minutes (final: 9 minutes)] were isolated by RT-PCR with degenerate primers using RNA from the anterior part of the axis of early neurula stage (stage 17) embryos and TA cloned into the pCRII vector (Invitrogen). Identity of the cloned fragments was confirmed by sequencing and comparison to the known vertebrate homologues (EMBL accession numbers are Pax2, Z97020 and shh, Z97019).

\section{In situ hybridization and immunohistochemistry}

In situ hybridization was performed as described by Oliver et al. (1996). When double staining was carried out medaka sal in situ hybridization was performed first. Acetylated tubulin was detected using a monoclonal antibody (Sigma \#T6793 1:1000 dilution). Secondary anti-mouse antibody coupled to alkaline phosphatase (Jackson ImmunoResearch, 1:200 dilution) was preabsorbed to medaka embryos. Phosphatase activity was detected using Fast Red (Boehringer Mannheim) as substrate. Stained embryos were briefly rinsed in methanol and mounted in $87 \%$ glycerol. Intensely stained embryos were embedded in Technovit 7100 (Kulzer), sectioned $(6 \mu \mathrm{m})$ and mounted in Entellan (Merck).

\section{RNA injections}

Capped RNA for injections was transcribed from linearized pSP64T (Krieg and Melton, 1984) plasmids using the SP6 mMESSAGE mMACHINE Kit (Ambion \#1340). Individual blastomeres of 2- to 4cell stage embryos were injected as described by Wittbrodt and Rosa (1994). Initially different dilutions of mRNA were injected at concentrations of 50,125 and $250 \mathrm{ng} / \mu \mathrm{l}(\mathrm{shh})$ and $2.5,5,10$ and $20 \mathrm{ng} / \mu \mathrm{l}$ (PKA*) respectively. Concentration dependent effects observed in injected embryos were comparable to those described previously in that they became increasingly severe with rising mRNA concentrations in the injection solution (Ekker et al., 1995b; Hammerschmidt et al., 1996). Due to moderate morphological effects in injected embryos, concentrations of $125 \mathrm{ng} / \mu \mathrm{l}(\mathrm{sh})$ and $2.5 \mathrm{ng} / \mu \mathrm{l}\left(\mathrm{PKA}^{*}\right)$ respectively were chosen for the injection experiments shown in Figs 5 and 6. Control injections using Xenopus EF1 $\alpha$ (Ambion) mRNA were performed at identical concentrations. All injections were monitored with RNA encoding $\beta$-galactosidase ( $5 \mathrm{ng} / \mu \mathrm{l}$ ), and subsequently a number of embryos were stained for lac $Z$ activity to visualize distribution and functional integrity of injected RNA (Joore et al., 1996).

\section{RESULTS}

\section{Isolation and analysis of the medaka sal gene}

Based on the comparison of known vertebrate (Hollemann et al., 1996; Ott et al., 1996) and Drosophila (Kühnlein et al., 1994) spalt sequences we designed degenerate oligonucleotides specific for regions conserved in these spalt homologues. PCR amplification from medaka genomic DNA resulted in a $2.5 \mathrm{~kb}$ fragment. This fragment was subsequently used to screen a late neurula stage (stage 18) medaka cDNA library. Sequence analysis of the longest cDNA clone of $4.5 \mathrm{~kb}$ revealed that it contained the complete $3^{\prime}$ untranslated region including a polyadenylation consensus but lacked an inframe AUG at the $5^{\prime}$ end. The sequence could be extended in the $5^{\prime}$ direction using sequence information from genomic DNA. So far we have not been able to further extend the known sequence by RACE-PCR. Sequence comparison with the known vertebrate spalt genes indicates that this clone lacks approximately 70 residues at the amino terminus.

The deduced amino acid sequence of medaka sal shows high overall similarity to the known vertebrate sal genes $(68 \%$ in comparison to mouse and $66 \%$ in comparison to Xenopus, respectively). Medaka sal encodes a protein that contains the three diagnostic double zinc finger domains and a single zinc finger of the $\mathrm{C} 2 \mathrm{H} 2$-motif, located adjacent to the second pair of zinc fingers, which are characteristic for all SAL homologues. The entire domain containing the zinc fingers of SAL is highly conserved between vertebrates and Drosophila respectively, and shows the typical H/C-link of SPALT zinc fingers as well as the diagnostic SAL-box (Kühnlein et al., 1994) in each second finger of the three double zinc finger regions (Fig. 1B). The high degree of conservation in the SALbox and the zinc fingers, which are required for sequence 
specific DNA binding (Barrio et al., 1996), suggest that all sal homologues recognize a highly conserved binding site. At the amino terminus SAL contains an additional single zinc finger of the C2HC-type characteristic for vertebrate SAL proteins (Hollemann et al., 1996). In summary these features identify medaka sal as a homologue of the Drosophila and the known vertebrate spalt genes (Kühnlein et al., 1994; Hollemann et al., 1996; Ott et al., 1996; Kohlhase et al., 1996).

\section{Spatiotemporal expression pattern of sal during embryogenesis}

In Drosophila, spalt functions downstream of the $h$ h signaling pathway (Sturtevant et al., 1997). To investigate whether medaka sal plays a similar role in vertebrates we first examined sal expression during early medaka embryogenesis at the time when $s h h$ is known to exert its patterning activity. Localization of medaka sal was examined by whole-mount in situ hybridization. Transcripts of sal are detected during development of the medaka embryo in the eye, the central nervous system, the excretory system, the somites and the developing fins.

Initially, sal expression is apparent at neurula stages (stage 18; for stages refer to Iwamatsu, 1994). Expression is detected in the anteroproximal part of the optic vesicle, in the optic stalk and the rostral optic vesicle prior to the formation of the multilayered retina (Fig. 2A,D). sal expression levels fade when lens embedding and invagination of the optic vesicle occur (stage 21, not shown).

From neurula stages onwards, sal is expressed in the neural tube along the entire anteroposterior (AP) axis of the developing central nervous system (CNS) (stage 18) (Fig. 2D,E). Later, during organogenesis (stage 26), expression in the CNS becomes restricted to single cell clusters in the trunk region of the neural tube, whereas anteriorly in the rhombencephalon sal transcripts are found bilaterally in each rhombomere (Fig. $2 \mathrm{C}, \mathrm{F})$. In rhombomere four $s a l$ is expressed in commissural cells which cross the midline.

In the dorsal part of the midbrain, sal is expressed in a sharp stripe (sal stripe) of two to three cell diameters width in the mid-hindbrain boundary (MHB). This

\section{$B$}

Numbering corresponds to fingers in A. expression domain is first seen at early neurula stages (stage 17) and persists in the isthmus until the constriction between midand hindbrain has formed and separates mes- and metencephalon (stage 26; Fig. 2).

Strong sal expression is detected in pro- and opistonephros from neurula stages onwards (Fig. 2). In the pronephros, the progenitor of the head nephros, high expression levels of sal are detected throughout embryogenesis. Concomitant with the development of the head nephros to a lymphatic organ, sal expression disappears (not shown). In the opistonephros, expression is restricted to the posteriormost somites and declines from posterior to anterior in a graded manner suggesting a transient requirement of sal expression in the formation of the opistonephros.

\section{Expression of sal in the trunk is restricted to somitic cells and neurons}

To analyze the expression of sal in the trunk in more detail transverse sections of whole-mount stained embryos where performed. Lower levels of sal expression were detected
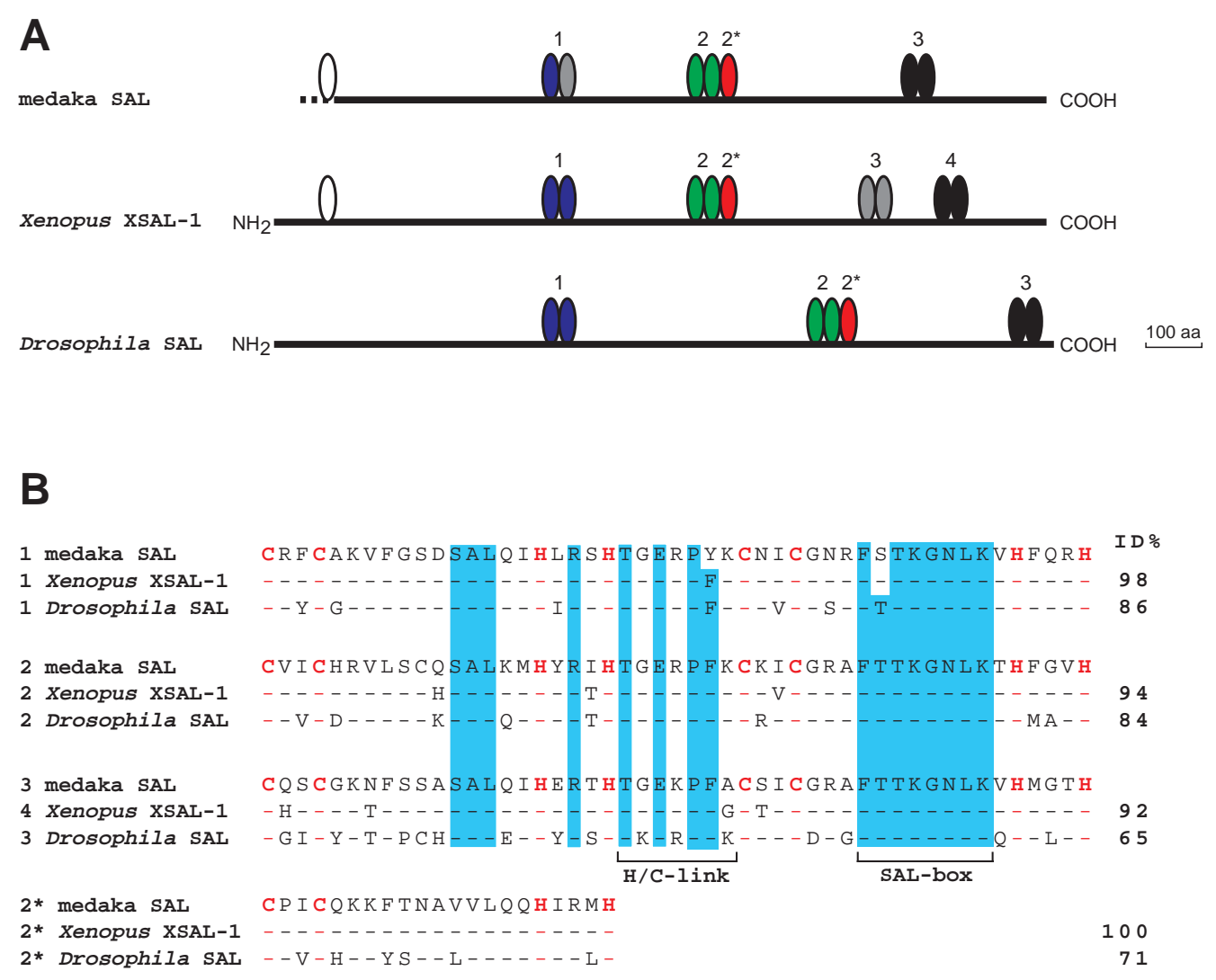

Fig. 1. Medaka sal is a vertebrate homologue of Drosophila spalt. (A) Diagrammatic representation of SAL proteins from medaka, Xenopus and Drosophila comparing relative positions of zinc finger motifs (ovals). Homologous fingers are in the same color; the single N-terminal C2HC motif, characteristic for vertebrate $\mathrm{SAL}$ proteins is in white. $2 *$ marks the single $\mathrm{C} 2 \mathrm{H} 2$ finger motif adjacent to the 2 nd double finger present in all SAL-type proteins. For the alternatively spliced 3rd double finger (grey) see Hollemann et al. (1996). (B) Sequence comparison of homologous zinc finger motifs. Zinc complexing cysteine and histidine residues are in red. Identical amino acid residues are shown as dashes, positions identical in all double finger motifs are highlighted in blue, the conserved H/C-link and the SAL-box are underlined. Sequence identity values correspond to the medaka fingers $1,2,3$, and $2 *$ respectively. 
A
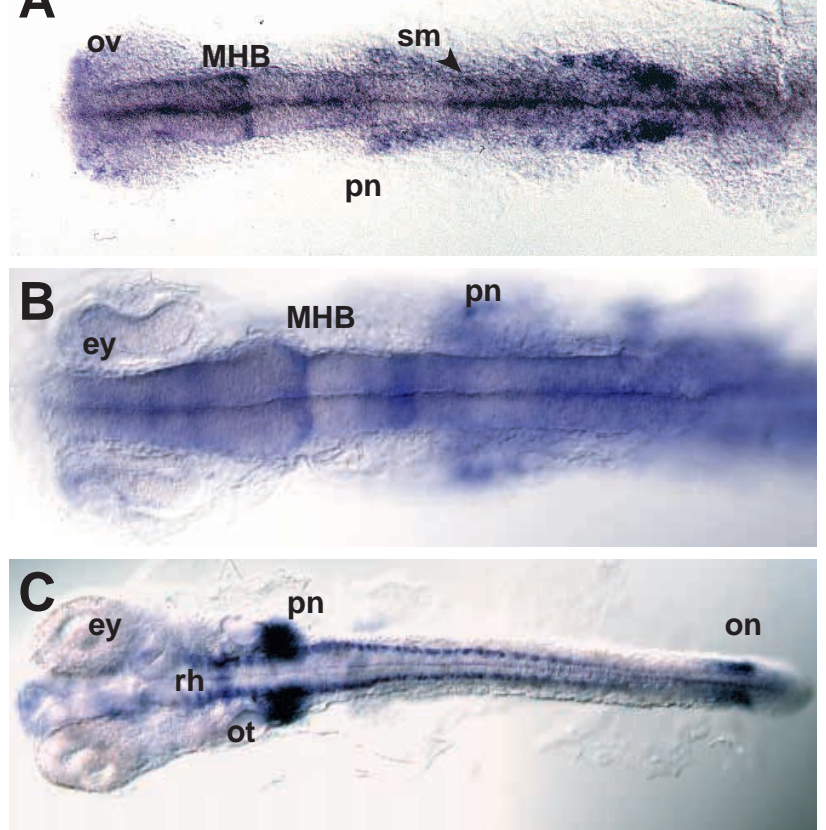

D

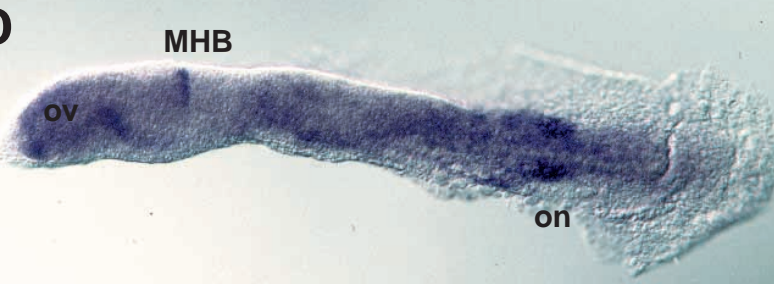

E
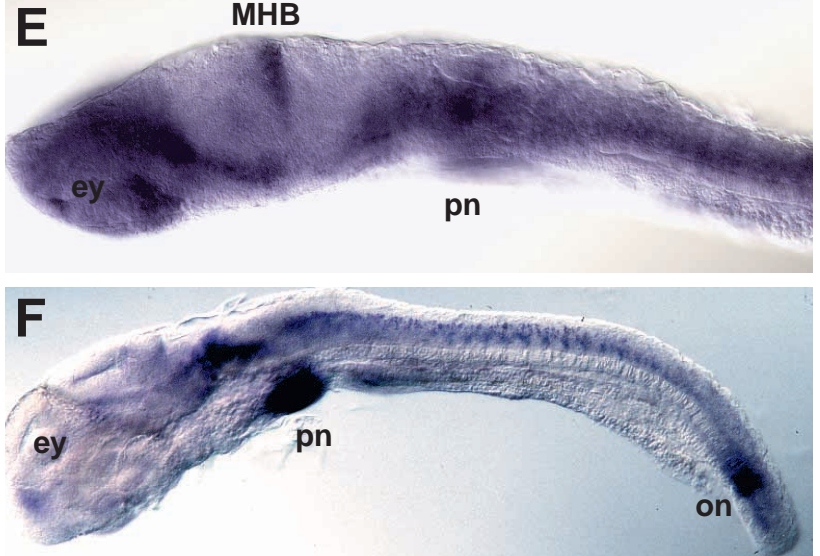

Fig. 2. Whole-mount in situ analysis during neurula stages and somitogenesis, anterior left, dorsal views (A-C), lateral views (D-F). (A,D) sal expression during neurula stages (stage 18) sal is expressed in the proximal part of the optic vesicle, in the neural tube along the anteroposterior axis and in the pro- and opistonephros. Note sharp expression domain in the dorsal part of the MHB (sal stripe). (B,E) Stage 24, intensity of sal expression in the sal stripe is increasing. Expression in the neural tube is confined to the ventralmost part. (C,F) Early organogenesis stages (stage 26). In rhombencephalon and spinal cord transcripts are detected in repeated clusters of 8-10 cells. In the rhombencephalon sal clusters are located in rhombomers 2-7. Prominent sal expression in pronephros and opistonephros. Note that expression in the opistonephros is confined to tubules in newly forming somites. ey, eye; MHB, mid-hindbrain boundary; on, opistonephros; ov, otic vesicle; pn, pronephros; rh, rhombencephalon.

medially in the forming somites, the region of the prospective sclerotome (Fig. 3A) which has been shown to be induced by long range signaling of a secreted $\mathrm{HH}$ family member (Fan and Tessier-Lavigne, 1994).

In neural structures sal expression is detected at neurula stages in the ventral third of the forming neural tube (stage 19), but is excluded from the ventralmost floorplate cells (Fig. 3). As neuronal differentiation proceeds during somitogenesis (stage 24), small bilateral clusters of sal-expressing cells are found in the ventral part of the neural tube (Fig. 3B). Here sal expression reflects the time course of neuronal differentiation, suggesting that sal transcripts are confined to differentiating neurons. To verify this, double labeling experiments were performed which combined in situ hybridization for sal and immunostaining of the neuronal axons (Chitnis and Kuwada, 1990) with an antibody directed against acetylated tubulin (Piperno and Fuller, 1985). sal-positive cells located ventrally in the neural tube project their tubulin-positive axons to the underlying somites, identifying these cells as motor neurons (Fig. 3C,D). Later, during organogenesis stages (stage 27), sal-expressing cell clusters are detected in intermediate and then in dorsal regions of the neural tube (Fig. 3E, 4E) representing interneurons and sensory neurons repectively. Taken together this shows that sal is expressed in neuronal cells, where its expression identifies differentiating neurons rather than a specific neuronal cell type.

\section{sal expression is confined to hedgehog signaling centers}

In vertebrates, hedgehog signaling is required for the proper pat- terning of the eye, neural tube, kidneys, somites and limb buds. Secreted SHH protein, diffusing from localized sources, mediates pattern formation within the $h h$ signaling center over short and long distances. In medaka, as reported for other vertebrates (Krauss et al., 1993; Riddle et al., 1993; Echelard et al., 1993; Roelink et al., 1994), shh is expressed in the notochord and subsequently in the ventral midline of the neural tube (Fig. 4A). Comparison of medaka sal and $s h$ expression shows that in the $h h$ signaling centers, the eye, the ventral part of the neural tube, the kidneys and somites, sal transcripts are found adjacent to shh expression (Fig. 4). In outbudding fins the expression domains of sal and $s h h$ are partially overlapping (not shown). At late neurula stages sal transcripts are found in the proximal part of the outbudding optic vesicle (Fig. 4C), which is known to be under the control of $h h$ signaling in vertebrates (Ekker et al., 1995b; Macdonald et al., 1995; Chiang et al., 1996). Transverse sections through the rostral head region reveal sal expression in the optic chiasm (Fig. 4D) and a faint expression in the more distal part of the optic vesicle (Fig. 4D). In the early neural tube sal expression is limited to the ventral region (Figs 3A, 4D) but excluded from the floorplate cells expressing shh (Figs 3A, 4A). This expression of $s a l$ is subsequently refined to differentiating neurons and reflects the patterning activity of SHH which mediates at least two phases of patterning. First, medial neural plate cells are converted to ventralized progenitors (expressing sal; Fig. 3A), which are then, in a second step induced directly by SHH to differentiate into (sal positive; Fig. 3B-D) motor neurons (Marti et al., 1995; Tanabe et al., 1995; Ericson et al., 1996). The absence of the second induction step leads to the 
induction of interneurons (Fig. 4E), located more dorsally, indicating that $\mathrm{SHH}$ acts as a morphogen in the neural tube (Ericson et al., 1996). The finding of sal expression at organogenesis stages in dorsally located sensory neurons (Fig. 3E) might be due to long range diffusion of an $H H$ signal through the cavity of the neural tube as suggested for patched in chicken (Marigo and Tabin, 1996).

The pronephros shows abundant sal expression throughout embryonic development (Figs 2, 4E), which is consistent with the suggested role for $s h h$ in kidney formation in higher vertebrates (Fig. 4E; Bitgood and McMahon, 1995; Chiang et al., 1996).

In the pectoral fin buds (not shown) and subsequently in the outgrowing pectoral fins, sal transcripts are localized in the posterior half of the distal progress zone (Fig. 4F), an area analogous to the zone of polarizing activity (ZPA) of higher vertebrates. Here shh is required for proper AP-patterning of the limb (Riddle et al., 1993; Chang, 1994).

Thus, in all tissues known to be patterned by $\mathrm{HH}$ activity, expression of sal and shh coincide temporally and spatially, suggesting that sal expression is activated in response to $\mathrm{HH}$ activity.

\section{Ectopic $\mathrm{HH}$ activity expands sal expression domains}

We have tested this inference using two complementary experimental strategies. First, we have examined the sal-inducing activity of $\mathrm{SHH}$ by injecting different amounts of shh mRNA (Krauss et al., 1993) into one blastomere at the 2-4 cell stage. Due to the limited stability of the RNA injected (data not shown) we focused our analysis on early effects and therefore analyzed embryos up to stage 22 . Functional expression of ectopic $s h h$ was monitored by the altered size and shape of the developing eyes (Fig. 5C,H) (Ekker et al., 1995b; Macdonald et al., 1995). Already moderate levels of ectopic SHH activity expanded the sal expression domains significantly. We did not, however, observe ectopic de novo expression isolated from the wild-type sal expression domains. Fig. 5C shows that in shh injected embryos sal expression covers the entire eye, which is consistent with the observation that $h h$ overexpression causes an expansion of proximal eye fates (Ekker et al., 1995b; Macdonald et al., 1995). In the neural tube and in the brain of injected embryos the ventral wild-type sal expression domain (Fig. 5B,D) expands towards the dorsal side (Fig. 5C,E), however, sal expression was never detected in the dorsal part of the mesencephalon (Fig. 5C). In the somites (Fig. 5D) and the pronephros (Fig. 5B), the mesodermal sal expression domains, shh injection causes a significant enlargement of the sal domains (Fig. 5C,E).

These results show that sal expression, which expands in $h h$ signaling centers in response to increased $\mathrm{HH}$ activity, can indeed be activated by $h h$ signaling. The finding that no de novo sal expression domains are observed, indicates that additional competence factors determine the response to ectopic $\mathrm{HH}$ activity which, in the case of sal, allow only an expansion of the wildtype expression domains.

\section{Ectopic PKA* activity reduces the size of the sal expression domains}

To further investigate the regulation of sal expression through the $h h$ signaling pathway we have assayed the effects of reduced $\mathrm{HH}$ activity. In Drosophila it was shown that protein
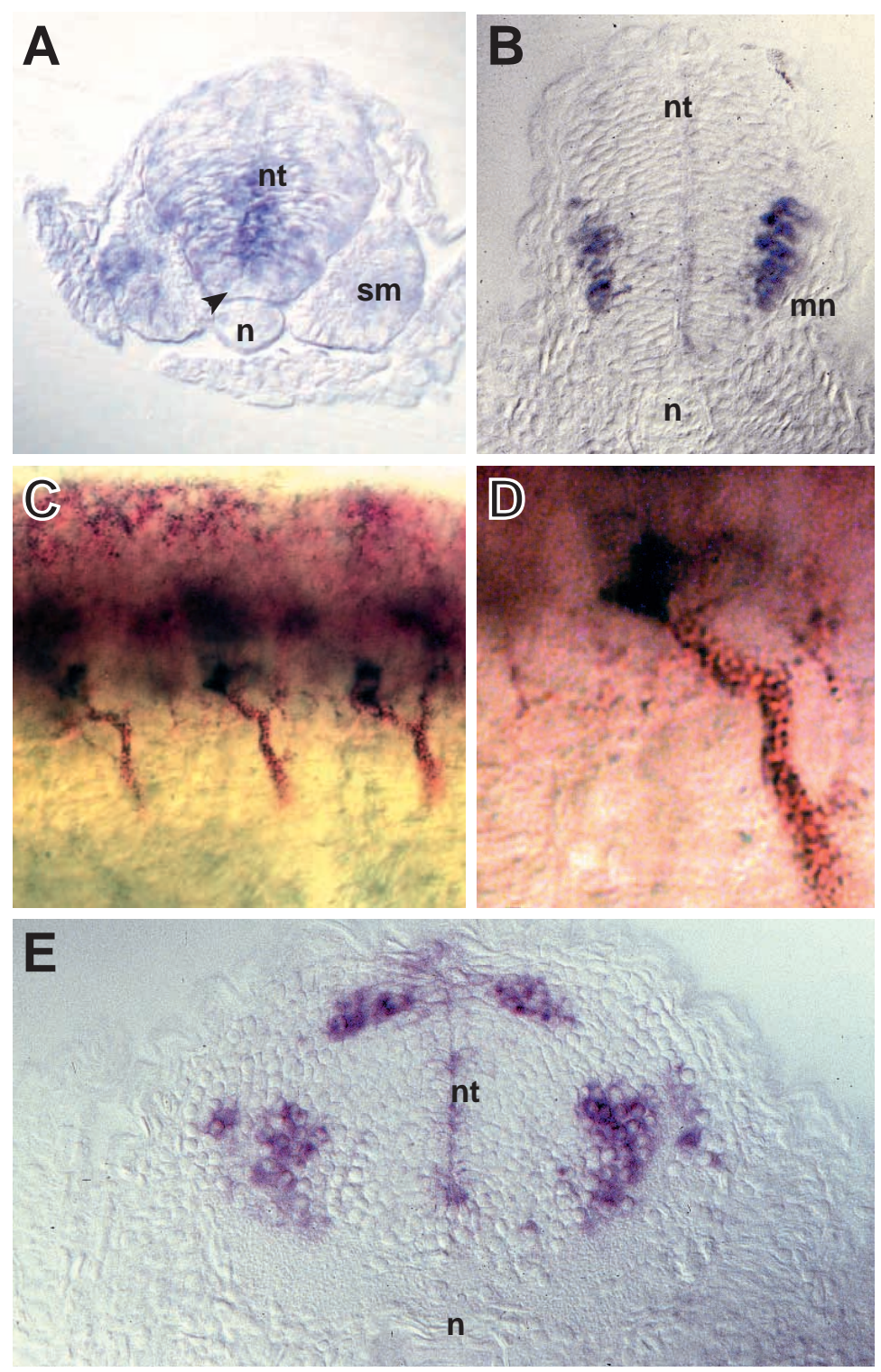

Fig. 3. Medaka sal expression is in the neural tube is confined to neurons. (A, B) Transverse sections through the trunk region of whole-mount stained embryos. (A) sal expression is restricted to the ventral third of the neural tube (stage 19). No sal expression is detected in the floorplate (arrowhead). In somites flanking the notochord faint sal expression is detected. (B) Ventral sal expression in the neural tube is restricted to ventral, bilateral clusters of 8-10 cells (stage 25). (C,D) Axons of primary motoneurons (red), visualized by an antibody directed against acetylated tubulin, project from sal-positive cell bodies (blue) to the underlying somites and identify sal-positive cells (in B) as motoneurons. (E) sal staining in a transverse section through the trunk region of an embryo at organogenesis stages (stage 27). In addition to ventral clusters of sal-positive cells in the neural tube staining becomes apparent at intermediate positions. mn, motoneurons; n, notochord; nt, neural tube; sm, somite; fp, floorplate 
kinase A (PKA) acts to suppress the expression of $h h$ target genes (Jiang and Struhl, 1995; Lepage et al., 1995; Li et al., 1995; Pan and Rubin, 1995; Strutt and Mlodzik, 1995). To interfere with $h h$ signaling we made use of a constitutively active form of the catalytic subunit of protein kinase A (Orellana and McKnight, 1992; PKA*) previously shown to antagonize known $h$ signaling activities in vertebrates (Hammerschmidt et al., 1996; Concordet et al., 1996).

The injection of PKA* mRNA causes fusion of the eyes (Fig. 6B,D), indicating that $\mathrm{PKA}^{*}$ activity is functional in antagonizing midline signaling mediated by $\mathrm{HH}$ activity as reported previously (Hammerschmidt et al., 1996; Concordet et al., 1996). While ectopic SHH was expanding the sal expression domains, the expression of $\mathrm{PKA}^{*}$ had the opposite effect. We observed a concentration dependent, strong reduction of sal expression in all $h h$ signaling centers. In the eye, minute traces of sal expression are detected (Fig. 6B). sal expression in the ventral CNS is almost eliminated upon PKA* injection (Fig. 6B). Only weak residual sal transcription is detected in the pronephros (Fig. 6B) and somites. Thus, sal expression is reduced in all sal expression domains confined to $h h$ signaling centers, when $h h$ signaling is antagonized by the expression of PKA*.

In addition to its expression in the known $h h$ signaling centers described so far, sal is also detected at organogenesis stages in dorsally located neurons within the neural tube and already at the neurula stage in a stripe at the midbrain hindbrain boundary (MHB; Fig. 4B). While the limited stability of the mRNA injected did not allow the investigation of the effect of ectopic shh and its antagonist PKA* respectively on dorsal sal expessing neurons, this issue could be addressed for the sal expression at the MHB.

\section{sal expression at the MHB responds to ectopic shh and its antagonist PKA*}

The MHB was previously identified as a patterning center required for the establishment of an anteriorposterior polarity in the midbrain (reviewed by Joyner,

Fig. 4. Medaka sal expression is confined to $h h$ signaling centers. (A) Lateral view of a stage 24 embryo (early somitogenesis) stained for medaka shh. shh is detected in prechordal mesoderm and along the AP-axis in the ventralmost part of the CNS (floorplate). More caudally expression is detected in the notochord. (B-F) sal expression. $(\mathrm{B}, \mathrm{C})$ sal is detected in proximal part of optic vesicle, in optic stalk and in optic chiasm, revealed by transverse sections at the level of the eye in stage 19 embryos (D). sal transcripts are detected in forming neural tube (arrowheads in B) restricted to its ventral part (D) and subsequently in differentiating neurons (E; see also Fig. 3). (B,E) sal expression is found in the mesodermally derived pronephros. (F) In outgrowing pectoral fins sal expression is confined to the posterior part. In addition to known $h h$ signaling centers sal is also expressed in the MHB (B,C) where no shh expression is detected (A). ey, eye; fp, floorplate; $\mathrm{MHB}$, mid-hindbrain boundary; n, notochord; nt, neural tube; oc, optic chiasm; os, optic stalk; ot, otic vesicle; ov, otic vesicle; pn, pronephros.
1996). shh or other members of the $h h$ family have not been identified as an essential signaling activity in this dorsal brain region. However, the sal stripe at the MHB clearly expands in response to ectopic shh expression (Fig. 5C). It expands from only a few cell diameters width in wild-type embryos (Fig. 5B) up to five fold in the AP direction in response to ectopic $\mathrm{HH}$ activity (Fig. $5 \mathrm{C})$. Conversely, inhibition of $h h$ signaling in $\mathrm{PKA}^{*}$ injected embryos, which efficiently suppresses sal transcription in all $h h$ signaling centers, also prevents the sal stripe expression at the MHB (Fig. 6B).
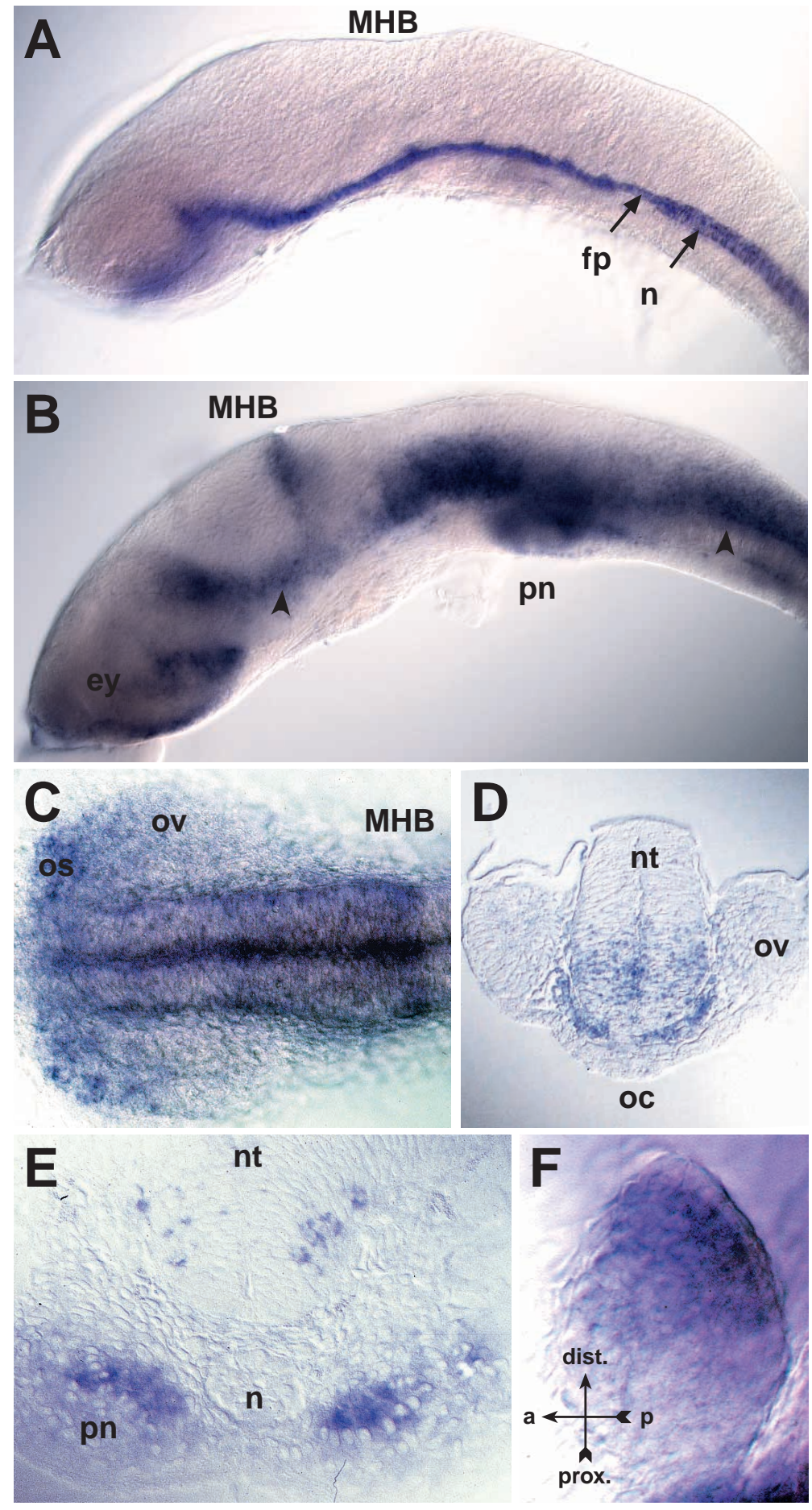


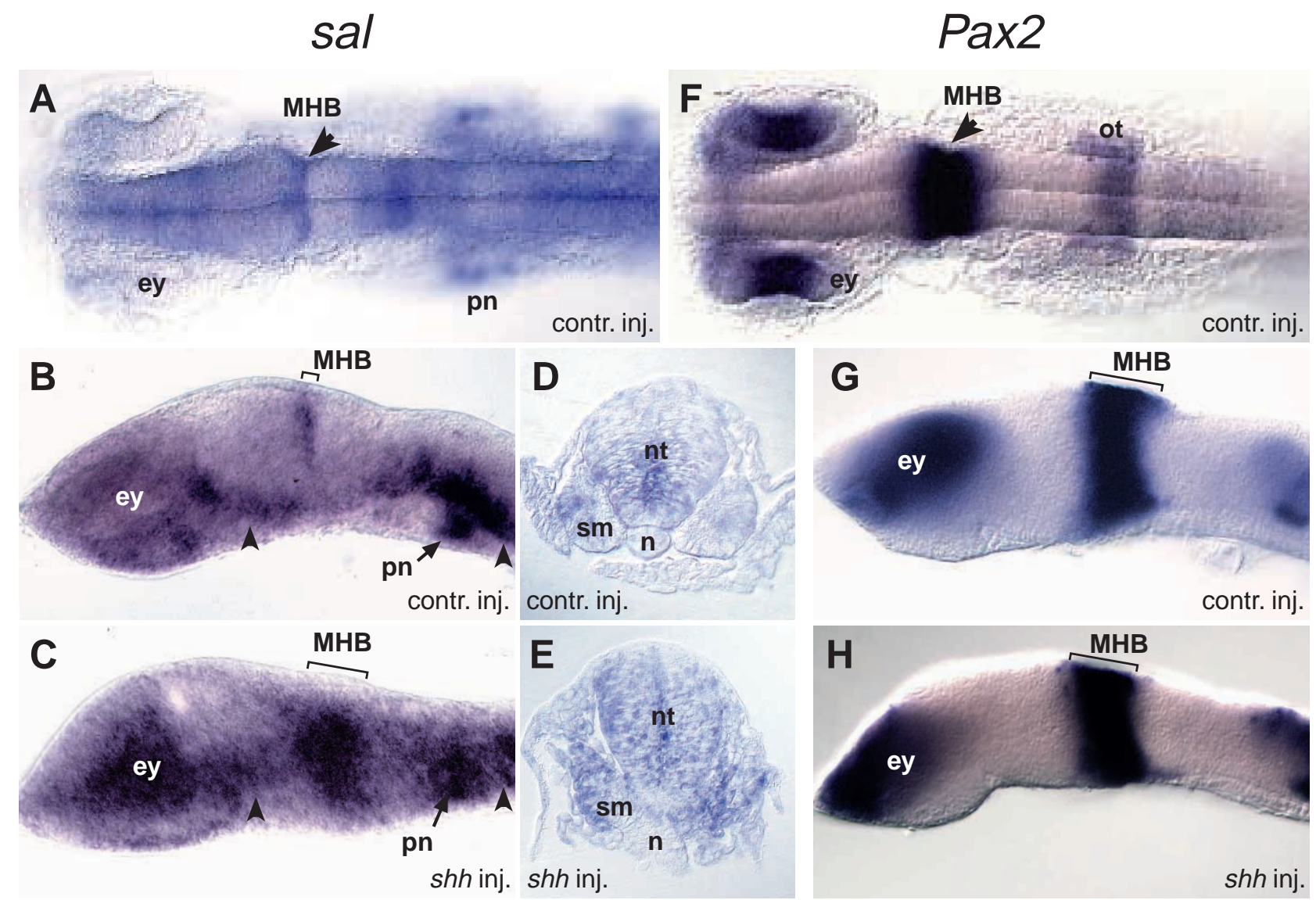

Fig. 5. sal expression domains adjacent to $s h$ expression are expanded by ectopic shh. Detection of sal (A-E) and Pax2 transcripts (F-H) at stage 22, anterior is left. Compare expression patterns in control (A,B,D,F,G; XEF1 $\alpha$, ambion) and shh injected (C,E,H) embryos. (A) sal stripe (arrowhead) in the MHB is within Pax2 expression domain (F). Faint expression in the eye, restricted to proximal structures (dorsal view). (B) Lateral view of same embryo. (C) Upon shh injection sal expression expands distally in eye, dorsally in neural tube (arrowhead) and in pronephros (arrow). sal stripe is expanded in AP-direction. Compare dimensions of sal stripe (bar) with the Pax2 domain in H. Cross sections of (D) control and (E) shh-injected embryos at the level of the spinal cord. sal expression in somites and neural tube is expanded. (F) Dorsal view shows Pax2 expression in entire MHB and in proximal parts of the eye. (G) Pax2 expression in control and (H) shh injected embryos. Pax2 is detected in entire eye. Dimensions of MHB expression domain are unaffected. Abbreviations: ey, eye; MHB, mid-hindbrain boundary; n, notochord; nt, neural tube; ot, otic vesicle; pn, pronephros; sm, somite.

As $h h$ signaling has not been found to be required for APpatterning in the dorsal midbrain, we asked whether the experimentally induced $\mathrm{HH}$ effect on sal stripe expression is an indirect one, such as an HH-induced overproliferation of the sal-expressing cells. To clarify this, we examined the expression domain of Pax2, a cellular marker for the size of the MHB area (Krauss et al., 1992; Brand et al., 1996). Figs 5H, 6D show that Pax 2 expression in the eye depends on $\mathrm{HH}$ activity, as reported previously. But neither ectopic shh nor PKA* significantly altered the dimension of the MHB Pax2 expression domain, indicating that the expansion of the sal stripe is not caused by cell proliferation events in response to ectopic HH activity. This suggests that sal expression in the MHB also depends directly on $h h$ signaling.

\section{DISCUSSION}

\section{$\boldsymbol{s a l}$ is a general target of $\boldsymbol{h} \boldsymbol{h}$ signaling}

Signaling by $h h$ and the vertebrate hedgehog gene family is a pat- terning mechanism involved in many different developmental processes in Drosophila and vertebrates. The secreted HH protein diffuses and binds to its receptor complex consisting of at least two multipass membrane spanning proteins, SMOOTHENED and PATCHED (Stone et al., 1996; Marigo et al., 1996a). The receptors patched in chicken and $p t c l$ in zebrafish, which are the vertebrate homologues of Drosophila patched, are expressed in an $h h$-dependent manner in $h h$ signaling centers adjacent to known sites of $h h$ expression (Marigo and Tabin, 1996; Concordet et al., 1996). In vertebrates, target genes that can mediate $h h$ patterning on the transcriptional level have been described only for a few signaling centers. Here we report the identification of a novel hedgehog target gene, the medaka gene sal. It encodes all features diagnostic for a DNA-binding zinc finger protein and thus, like its Drosophila homologue, it is likely to act as a transcriptional regulator. In contrast to other transcription factors which act as $h h$ targets in a subset of the $h h$ signaling centers, sal expression is found in all known $h h$ signaling centers. sal expression expands in response to increased $\mathrm{HH}$ activity and is repressed in response to PKA* and thus, it 
Fig. 6. Suppression of $h h$ signaling prevents sal expression. Detection of sal $(\mathrm{A}, \mathrm{B})$ and Pax2 transcripts (C,D) in lateral views of stage 22 embryos. Compare expression patterns of control (XEF1 $\alpha$, ambion; A,C) and PKA* injected (B,D) embryos.

(A) Wt sal expression in the eye and ventral neural tube (arrowheads) as well as in the pronephros (arrow) is strongly reduced or completely absent in PKA* injected embryos (B). No sal stripe expression is detectable in the MHB. (C) Pax2 expression in control and (D) PKA* injected embryos. Expression in the eye is suppressed by PKA* whereas expression in the MHB is unaffected. Axial mesoderm (asterisk) underlying the neural tube is extended towards anterior upon PKA* injection. Abbreviations as in Fig. 5
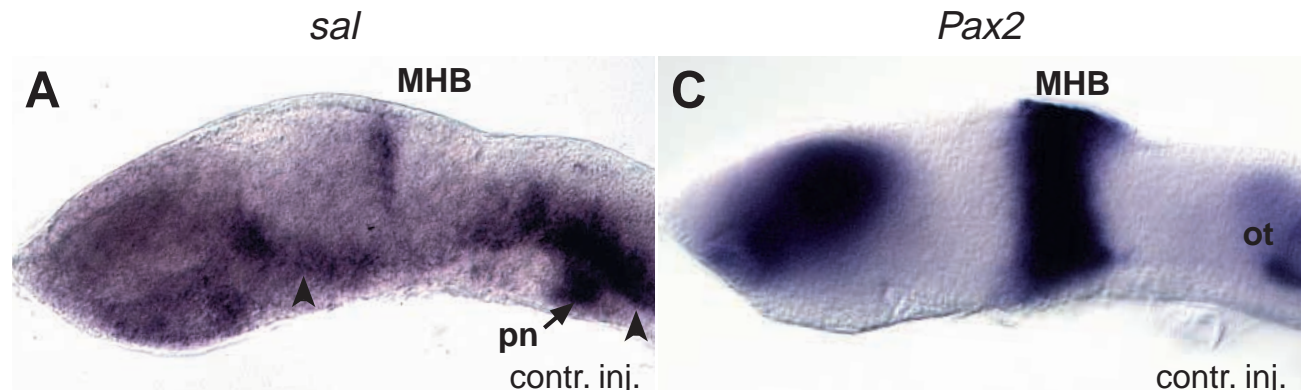

B

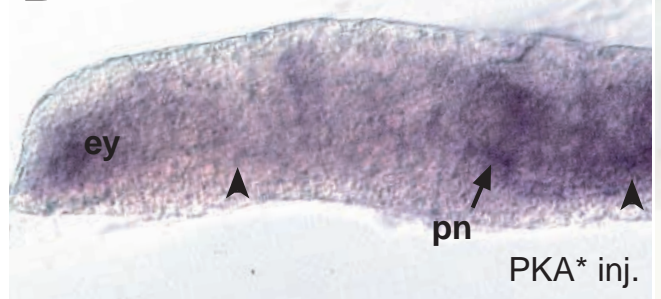

D

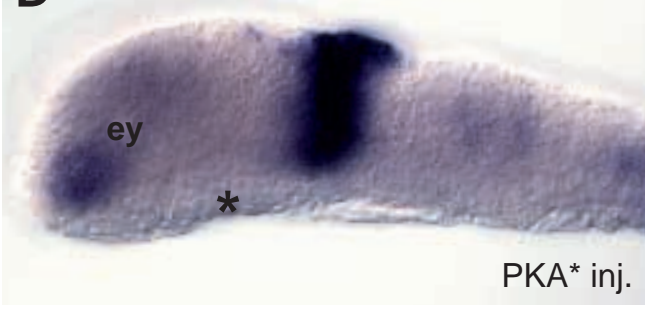

appears to be a general target of $h h$ in the medaka fish. The finding of a competence area surrounding the wild-type sal expression domains provides some evidence for an immediate control of sal by $h h$ although, at the moment, we cannot decide whether this control by $h h$ signaling is direct or mediated.

sal expression is observed in all known $h h$ signaling centers including the optic stalk and anterior optic vesicle where shh acts in proximodistal pattering. This domain of sal expression is expanded upon ectopic HH activity whereas blocking of $h h$ signaling by $\mathrm{PKA}^{*}$ prevents its expression. In the proximal part of the eye, $n k 2.2$ (Barth and Wilson, 1995) and Pax2 have been reported as $h h$ target genes (Ekker et al., 1995b; Macdonald et al., 1995). Unlike sal, which is $h h$ dependent in all $h h$ signaling centers, the expression of $n k 2.2$ is limited to the proximal parts of the developing eye and the ventral part of the neural tube. $\operatorname{Pax} 2$ shows a responsiveness to $h h$ signaling only in the eye expression domain (Ekker et al., 1995b; Macdonald et al., 1995 and our results), while its expression in the MHB and at other locations does not depend on $h h$ signaling.

sal expression in the neural tube, somites and limb buds is reminiscent of the expression pattern of the zinc-finger transcription factor GLI in chick (Marigo et al., 1996b) which was shown to be controlled by shh in the limb. In the neural tube SHH exerts its patterning activity in two phases. First, naive neural plate cells are converted to ventralized progenitors (positive for sal and GLI). In a second step SHH emanating from the floor plate directly induces motoneurons. Peak concentrations of $\mathrm{SHH}$, which acts as a morphogen in the neural tube, induce exclusively the expression of floor plate markers (e.g. HNF3ß/axial), while slightly lower concentrations induce expression of motoneuron specific markers and intermediate concentrations induce the expression of interneuron markers (Ericson et al., 1996). This patterning activity is reflected by the expression of sal which is initially present over the entire ventral part of the neural tube (excluded from the floorplate), but is subsequently refined to motoneurons and is later also detected in interneurons. In contrast, GLI is expressed in the ventral neural tube without further refinement (Marigo et al., 1996b). Late dorsal sal expression in sensory neurons could likely represent a long range induction through SHH secreted from the floorplate diffusing up through the lumen of the neural tube, as suggested for patched in chicken (Marigo and Tabin, 1996). This induction might also be due to the activity of other members of the $h h$ family expressed in dorsal regions of the spinal chord like banded hh in Xenopus (Ekker et al., 1995a). Alternatively, dorsally expressed BMP4 (Liem et al., 1995), the vertebrate homologue of Drosophila dpp, which activates spalt in the Drosophila wing imaginal disc (Lecuit et al., 1996; Nellen et al., 1996; de Celis et al., 1996), may be required for late sal transcription in sensory neurons.

Ectopic expression of $s h h$ leads to an expansion of the wildtype sal expression domains and does not cause de novo expression of sal. In contrast the $h h$ target genes zp50 (Hauptmann and Gerster, 1996) and axial (Strähle et al., 1993) are expressed at ectopic locations upon $s h$ injection, indicating different competences in the $h h$ response. In $s h h$ injected embryos zp50 is expressed in ectopic domains with a drastic loss of wt expression domains (Hauptmann and Gerster, 1996). axial, like sal, upon shh injection exhibits a widening of its ventral neural tube expression. In addition ectopic de novo expression was detected in the MHB region (Krauss et al., 1993) indicating a competence for $h h$ signaling. The widening of the sal expression domains upon $s h$ injection is best explained by assuming that the ectopic shh adds to the endogenous concentration of $\mathrm{HH}$ and thereby increases the critical concentration thresholds of $\mathrm{HH}$ in the signaling centers. The finding that no de novo sal expression domains are observed indicates that additional competence factors determine the response to ectopic $\mathrm{HH}$ activity, which allow sal activation only in the competent tissue. Taken together, these results indicate that sal is indeed a specific and general target of $h h$ signaling.

\section{Does the sal stripe define a new $h h$ signaling center?}

In addition to the known $h h$ signaling centers we found that the sal expression domain at the MHB expands in response to $h h$ signaling. Ectopic transplantation studies in chick and quail have demonstrated that the MHB, the junction of mid- and hindbrain, acts as an organizer that has a strong polarizing 
activity on midbrain and hindbrain (Bally Cuif et al., 1992; Otero et al., 1993). So far the expression pattern and functional analysis of known hedgehog family members is consistent with a role in dorsoventral patterning of the CNS. However, no member of the $h h$ family has been shown to be expressed in an area around the MHB nor has it been shown that $\mathrm{HH}$ activity is involved in AP-patterning at the MHB.

Our results, however, indicate that the sal stripe expression domain responds directly to ectopic $h h$ signaling. This finding and the notion that $s a l$ is expressed in all known $h h$ signaling centers, suggest that the sal stripe expression depends on nondetectable amounts of known members of the $\mathrm{HH}$ family of secreted molecules or on an unknown member of the family. This argument is consistent with the finding that ectopic PKA* represses sal expression in all these domains, including the MHB.

A distinct $\mathrm{HH}$ competence region could be established by cell migration events in the neural keel at the level of the MHB. In this view, a cluster of cells which is initially in contact with $\mathrm{HH}$ emanating from the ventral midline could migrate dorsally in a highly coordinated manner and give rise to the MHB region expressing sal in response to the initial SHH activity in the ventral midline. We have not observed dorsal migration of sal-expressing cells within the corresponding region and thus, such a mechanism underlying HH-dependent sal expression at the wild-type MHB appears unlikely.

A second possibility that may account for $\mathrm{HH}$-dependent sal expression at the MHB is a localized dorsal competence responding to long range shh signaling after its ventral expression in the neural tube. The expression pattern of patched, one of the receptor components of the $h h$ signaling cascade in the midbrain-hindbrain boundary of chicken (Marigo and Tabin, 1996), is consistent with this proposal. Additional evidence in support of this model is provided by the observation of ectopic MHB expression of another $h h$ target gene, axial (Strähle et al., 1993) in response to ectopic shh (Krauss et al., 1993). axial is normally expressed at the ventral midline of the neural tube and in the diencephalic-mesencepahlic boundary (Strähle et al., 1993). Upon injection of $s h h$ mRNA, axial is ectopically expressed in the region of the MHB (Krauss et al., 1993) indicating a localized competence to $h h$ signaling in the MHB. In contrast to $s a l$, which is detected by the early neurula stage in the dorsal part of the MHB area, the dorsal patched expression seems to evolve from a more ventrally localized expression domain. Marigo and Tabin (1996) suggest that patched expression in the MHB is likely to represent a long range action of the ventral SHH signal, facilitated through diffusion of SHH in the lumen of the neural tube. At the time when the sal stripe is first detected in the prospective MHB, a neural tube has not yet formed because of fish secondary neurulation (Papan and Campos-Ortega, 1994). This property of fish development makes it unlikely that long range diffusion of ventral SHH through the lumen of the neural tube would be the cause for the sal stripe expression, but we cannot rule out long range signaling through the neural keel itself.

A third scenario, based on the proposal of a local HH competence in the MHB, involves an additional novel source of $h h$. This proposes a novel member of the family, as none of the $h h$ family members described so far has been shown to be expressed dorsally in the MHB. This could be induced by FGF8, a diffusible molecule prominently involved in the pat- terning at the MHB (Crossley et al., 1996a) and a member of the fibroblast growth factor family of secreted factors. FGF8 is required for AP patterning of the midbrain and it also mediates AP patterning in the developing limb where it induces $s h h$ expression in the ZPA (Crossley et al., 1996b). This link between the two signaling pathways in the limb and the dependence of the MHB sal stripe on $h h$ signaling consistently argues for an unknown member of the $h h$ family to be involved in the sal induction and pattern organizing activity at the MHB.

We want to acknowledge H. Jäckle, R. P. Kühnlein and M. Gonzalez-Gaitan for continuous discussions and helpful suggestions, M. Hammerschmidt for providing the PKA* construct, E. Abouheif and J. Forjanic for critical reading of the manuscript; Manuela Kurth and Annette Krone for excellent technical assistance. This work was supported by grants from the SFB271 (J. W., R. S.), the Boehringer Ingelheim Foundation (R. K.) and by the Swiss National Science Foundation and the Ciba-Geigy-Jubiläums-Stiftung (F. L.).

\section{REFERENCES}

Bally Cuif, L., Alvarado Mallart, R. M., Darnell, D. K. and Wassef, M. (1992). Relationship between Wnt-1 and En-2 expression domains during early development of normal and ectopic met-mesencephalon. Development 115, 999-1009.

Barrio, R., Shea, M. J., Carulli, J., Lipkow, K., Gaul, U., Frommer, G., Schuh, R., Jackle, H. and Kafatos, F. C. (1996). The spalt-related gene of Drosophila melanogaster is a member of an ancient gene family, defined by the adjacent, region-specific homeotic gene spalt. Dev. Genes Evolution 206, 315-325.

Barth, K. A. and Wilson, S. W. (1995). Expression of zebrafish nk2.2 is influenced by sonic hedgehog/vertebrate hedgehog-1 and demarcates a zone of neuronal differentiation in the embryonic forebrain. Development 121, $1755-1768$.

Bitgood, M. J. and McMahon, A. P. (1995). Hedgehog and BMP genes are coexpressed at many diverse sites of cell-cell interaction in the mouse embryo. Dev. Biol. 172, 126-138.

Brand, M., Heisenberg, C.-P., Jiang, Y.-J., Beuchle, D., Lun, K., vanEeden, F. J. M., Furutani-Seiki, M., Granato, M., Haffter, P., Hammerschmidt, M., Kane, D. A., Kelsh, R. N., Mullins, M. C., Odenthal, J. and NüssleinVolhard, C. (1996). Mutations in zebrafish genes affecting the formation of the boundary between midbrain and hindbrain. Development 123, 179-190.

Chang, D. T. (1994). Products, genetic linkage and limb patterning activity of a murine hedgehog gene. Development 120, 3339-3353.

Chiang, C., Litingtung, Y., Lee, E., Young, K. E., Corden, J. L., Westphal, H. and Beachy, P. A. (1996). Cyclopia and defective axial patterning in mice lacking Sonic Hedgehog gene function. Nature 383, 407-413.

Chitnis, A. B. and Kuwada, J. Y. (1990). Axonogenesis in the brain of zebrafish embryos. J. Neurosci. 10, 1892-1905.

Concordet, J. P., Lewis, K. E., Moore, J. W., Goodrich, L. V., Johnson, R. L., Scott, M. P. and Ingham, P. W. (1996). Spatial regulation of a zebrafish patched homologue reflects roles of sonic hedgehog and protein kinase A in neural tube and somite patterning. Development 122, 2835-2846.

Crossley, P. H., Martinez, S. and Martin, G. R. (1996a). Midbrain development induced by FGF8 in the chick embryo. Nature 380, 66-68.

Crossley, P. H., Minowada, G., MacArthur, C. A. and Martin, G. R. (1996b). Roles for FGF8 in the induction, initiation, and maintenance of chick limb development. Cell 84, 127-136.

de Celis, J. F., Barrio, R. and Kafatos, F. C. (1996). A gene complex acting downstream of dpp in Drosophila wing morphogenesis. Nature 381, 421424.

Echelard, Y., Epstein, D. J., St-Jacques, B., Shen, L., Mohler, J., McMahon, J. A. and McMahon, A. P. (1993). Sonic hedgehog, a member of a family of putative signaling molecules, is implicated in the regulation of CNS polarity. Cell 75, 1417-30.

Ekker, S. C., McGrew, L. L., Lai, C.-J., Lee, J. J., v. Kessler, D. P., Moon, R. T. and Beachy, P. A. (1995a). Distinct expression and shared activities of members of the hegehog gene family of Xenopus laevis. Development 121, 2337-2347. 
Ekker, S. C., Ungar, A. R., Greenstein, P., v. Kessler, D., Porter, J. A., Moon, R. T. and Beachy, P. A. (1995b). Patterning activities of vertebrate hedgehog proteins in the developing eye and brain. Curr. Biol. 5, 944-955.

Ericson, J., Morton, S., Kawakami, A., Roelink, H. and Jessel, T. M. (1996). Two critical periods of Sonic Hedgehog signalling required for the specification of notor neuron identity. Cell 87, 661-673.

Fan, C.-M., Porter, J. A., Chiang, C., Chang, D. T., Beachy, P. A. and Tessier-Lavigne, M. (1995). Long-range sclerotome induction by sonic hedgehog: Direct role of the amino-terminal cleavage product and modulation by the cyclic AMP signalling pathway. Cell 81, 457-465.

Fan, C.-M. and Tessier-Lavigne, M. (1994). Patterning of mammalian somites by surface ectoderm and notochord: Evidence for sclerotome induction by a hedgehog homolog. Cell 79, 1175-1186.

Hammerschmidt, M., Bitgood, M. J. and McMahon, A. P. (1996). Protein kinase $\mathrm{A}$ is a common negative regulator of Hedgehog signalling in the vertebrate embryo. Genes Dev. 10, 647-658.

Hammerschmidt, M., Brook, A. and McMahon, A. P. (1997). The world according to hedgehog. Trends Genet. 13, 14-21.

Hauptmann, G. and Gerster, T. (1996). Complex expression of the zp-50 pou gene in the embryonic zebrafish brain is altered by overexpression of sonic hedgehog. Development 122, 1769-1780.

Hollemann, T., Schuh, R., Pieler, T. and Stick, R. (1996). Xenopus Xsal-1, a vertebrate homolog of the region specific homeotic gene spalt of Drosophila. Mech. Dev. 55, 19-32.

Iwamatsu, T. (1994). Stages of normal development in the medaka Oryzias latipes. Zoo. Sci. 11, 825-839.

Jiang, J. and Struhl, G. (1995). Protein kinase A and hedgehog signaling in Drosophila limb development. Cell 80, 563-72.

Johnson, R. L., Laufer, E., Riddle, R. D. and Tabin, C. (1994). Ectopic expression of sonic hedgehog alters dorsal-ventral patterning of somites. Cell 79, 1165-1173.

Joore, J., Fasciana, C., Speksnijder, J. E., Kruijer, W., Destrée, O. H. J., v. d. Eijnden-v. Raaij, A. J. M., de Laat, S. W. and Zirkovic, D. (1996). Effects of retinoic acid on the expression of retinoic acid receptors during zebrafish embryogenesis. Mech. Dev. 55, 3-18.

Joyner, L. J. (1996). Engrailed, Wnt and Pax genes regulate midbrainhindbrain development. Trends Genet. 12, 15-19.

Kohlhase, J., Schuh, R., Dowe, G., Kühnlein, R. P., Jäckle, H., Schroeder, B., Schulz-Schaeffer, W., Kretzschmar, H. A., Köhler, A., Müller, U., Raab-Vetter, M., Burkhardt, E., Engel, W. and Stick, R. (1996). Isolation, characterization and organ-specific expression of two novel human zinc finger genes related to spalt of Drosophila. Genomics 38, 291-298.

Krauss, S., Concordet, J.-P. and Ingham, P. W. (1993). A functionally conserved homolog of the Drosophila segment polarity gene hh is expressed in tissues with polarizing activity in zebrafish embryos. Cell 75, 1431-1444.

Krauss, S., Maden, M., Holder, N. and Wilson, S. W. (1992). Zebrafish pax $[\mathrm{b}]$ is involved in the formation of the midbrain-hindbrain boundary. Nature 360, 87-89.

Krieg, P. A. and Melton, D. A. (1984). Functional messenger RNAs are produced by SP6 in vitro transcription of cloned cDNAs. Nucl. Acids Res. 12, 7057-7070

Kühnlein, R. P., Frommer, G., Friedrich, M., Gonzalez-Gaitan, M., Weber, A., Wagner-Bernholz, J. F., Gehring, W. J., Jäckle, H. and Schuh, R. (1994). Spalt encodes an evolutionary conserved zinc finger protein of novel structure which provides homeotic gene function in the head and tail region of the Drosophila embryo. ЕMBO J. 13, 168-179.

Lawrence, P. A. and Struhl, G. (1996). Morphogens, compartments, and pattern: lessons from Drosophila? Cell 85, 951-961.

Lecuit, T., Brook, W. J., Ng, M., Calleja, M., Sun, H. and Cohen, S. M. (1996). Two distinct mechanisms for long-range patterning by Decapentaplegic in the Drosophila wing. Nature 381, 387-393.

Lee, J. J., von Kessler, D. P., Parks, S. and Beachy, P. A. (1992). Secretion and localized transcription suggests a role in positional signalling for products of the segmentation gene hedgehog. Cell 70, 777-789.

Lepage, T., Cohen, S. M., Diaz-Benjumea, F. J. and Parkhurst, S. M. (1995). Signal transduction by cAMP-dependent protein kinase A in Drosophila limb patterning. Nature 373, 711-5.

Li, W., Ohlmeyer, J. T., Lane, M. E. and Kalderon, D. (1995). Function of protein kinase A in hedgehog signal transduction and Drosophila imaginal disc development. Cell 80, 553-62.

Liem, K. F., Jr., Tremml, G., Roelink, H. and Jessell, T. M. (1995). Dorsal differentiation of neural plate cells induced by BMP-mediated signals from epidermal ectoderm. Cell 82, 969-79.

López-Martinez, A., Chang, D. T., Chiang, C., Porter, J. A., Ros, M. A.,
Simandl, B. K., Beachy, P. A. and Fallon, J. F. (1995). Limb-patterning activity and restricted posterior localization of the amino-terinal product of Sonic hedgehog cleavage. Curr. Biol. 5, 791-796.

Macdonald, R., Barth, K. A., Xu, Q., Holder, N., Mikkola, I. and Wilson, S. W. (1995). Midline signalling is required for Pax gene regulation and patterning of the eyes. Development 121, 3267-3278.

Marigo, V., Davey, R. A., Zuo, Y., Cunningham, J. M. and Tabin, C. J. (1996a). Biochemical evidence that Patched is the Hedgehog receptor. Nature 384, 176-179.

Marigo, V., Johnson, R. L., Vortkamp, A. and Tabin, C. J. (1996b). Sonic hedgehog differentially regulates expression of Gli and Gli3 during limb development. Dev. Biol. 180, 273-283.

Marigo, V. and Tabin, C. J. (1996). Regulation of patched by sonic hedgehog in the developing neural tube. Proc. Natl. Acad. Sci. USA 93, 9346-9351.

Marti, E., Bumcrot, D. A., Takada, R. and McMahon, A. P. (1995). Requirement of $19 \mathrm{~K}$ form of sonic hedgehog for induction of distinct ventral cell types in CNS explants. Nature 375, 322-325.

Nellen, D., Burke, R., Struhl, G. and Basler, K. (1996). Direct and long-range action of a DPP morphogen gradient. Cell 85, 357-368.

Oliver, G., Loosli, F., Köster, R., Wittbrodt, J. and Gruss, P. (1996). Ectopic lens induction in fish in response to the murine homeobox gene Six3. Mech. Dev. 60, 233-239.

Orellana, S. A. and McKnight, S. (1992). Mutations in the catalytic subunit of cAMP-dependent proein kinase results in unregulated biological activity. Proc. Natl. Acad. Sci. USA 89, 4726-4730.

Otero, R. A., Sotelo, C. and Alvarado Mallart, R. M. (1993). Chick-quail chimeras with partial cerebellar grafts an analysis of the origin and migration of cerebellar cells. J. Comp. Neurol. 333, 597-615.

Ott, T., Kaestner, K. H., Monaghan, A. P. and Schütz, G. (1996). The mouse homolog of the region specific homeotic gene spalt of Drosophila is expressed in the developing nervous system and in mesoderm-derived structures. Mech. Dev. 56, 117-128.

Pan, D. and Rubin, G. M. (1995). cAMP-dependent protein kinase and hedgehog act antagonistically in regulating decapentaplegic transcription in Drosophila imaginal discs. Cell 80, 543-552.

Papan, C. and Campos-Ortega, J. A. (1994). On the formation of the neural keel and neural tube in the zebrafish Danio (Brachydanio) rerio. Rouxs Archiv. Dev. Biol. 203, 178-186.

Piperno, G. and Fuller, M. T. (1985). Monoclonal antibodies specific for an acetylated form of alpha-tubulin recognize the antigen in cilia and flagella from a variety of organisms. J.Cell Biol. 101, 2085-2094.

Riddle, R. D., Johnson, R. L., Laufer, E. and Tabin, C. (1993). Sonic Hedgehog mediates the polarizing activity of the ZPA. Cell 75, 1401-1416.

Roelink, H., Augsburger, A., Heemskerk, J., Korzh, V., Norlin, S., Ruiz i Altaba, A., Tanabe, Y., Placzek, M., Edlund, T., Jessell, T. M. and et al. (1994). Floor plate and motor neuron induction by vhh-1, a vertebrate homolog of hedgehog expressed by the notochord. Cell 76, 761-75.

Stone, D. M., Hynes, M., Armanini, M., Swanson, T. A., Gu, Q., Johnson, R. L., Scott, M. P., Pennica, D., Goddard, A., Phillips, H., Noll, M., Hooper, J. E., de Sauvage, F. and Rosenthal, A. (1996). The tumour-suppressor gene patched encodes a candidate receptor for sonic hedgehog. Nature 384, 129-134.

Strähle, U., Blader, P., Henrique, D. and Ingham, P. W. (1993). Axial, a zebrafish gene expressed along the developing body axis, shows altered expression in cyclops mutant embryos. Genes Dev.t 7, 1436-1446.

Strutt, D. I. and Mlodzik, M. (1995). Ommatidial polarity in the Drosophila eye is determined by the direction of furrow progression and local interactions. Development 121, 4247-4256.

Sturtevant, M. A., Biehs, B., Marin, E. and Bier, E. (1997). The spalt gene links the A/P compartment boundary to a linear adult structure in the Drosophila wing. Development 124, 21-32.

Tabata, T. and Kornberg, T. B. (1994). Hedgehog is a signalling protein with a key role in patterning Drosophila imaginal discs. Cell 76, 89-102.

Tanabe, Y., Roelink, H. and Jessell, T. M. (1995). Induction of motor neurons by sonic hedgehog is independent of floor plate differentiation. Curr. Biol. 5, 651-658.

Wittbrodt, J. and Rosa, F. M. (1994). Disruption of mesoderm and axis formation in fish by ectopic expression of activin variants: the role of maternal activin. Genes Dev. 8, 1448-1462.

Zardoya, R., Abouheif, E. and Meyer, A. (1996). Evolutionary analyses of hedgehog and Hoxd-10 genes in fish species closely related to the zebrafish. Proc. Natl. Acad. Sci. USA 93, 13036-13041. 